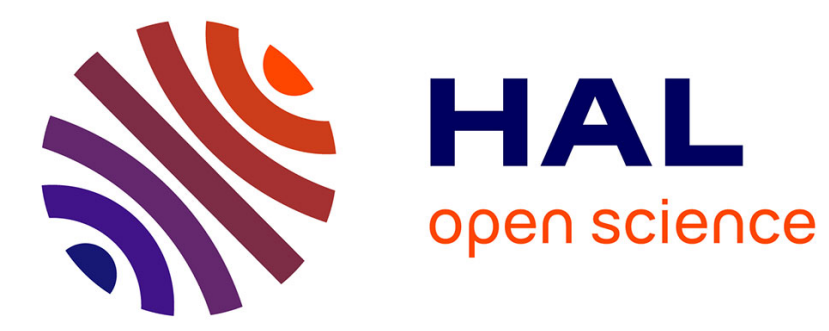

\title{
Optimization of the rectification factor of radiative thermal diodes based on two phase-change materials
}

\author{
Suraju Olawale Kasali, Jose Ordonez-Miranda, Karl Joulain
}

\section{To cite this version:}

Suraju Olawale Kasali, Jose Ordonez-Miranda, Karl Joulain. Optimization of the rectification factor of radiative thermal diodes based on two phase-change materials. International Journal of Heat and Mass Transfer, 2020, 154, pp.119739. 10.1016/j.ijheatmasstransfer.2020.119739 . hal-03017599

\author{
HAL Id: hal-03017599 \\ https://hal.science/hal-03017599
}

Submitted on 21 Nov 2020

HAL is a multi-disciplinary open access archive for the deposit and dissemination of scientific research documents, whether they are published or not. The documents may come from teaching and research institutions in France or abroad, or from public or private research centers.
L'archive ouverte pluridisciplinaire HAL, est destinée au dépôt et à la diffusion de documents scientifiques de niveau recherche, publiés ou non, émanant des établissements d'enseignement et de recherche français ou étrangers, des laboratoires publics ou privés. 


\title{
Optimization of the rectification factor of radiative thermal diodes based on two phase-change materials
}

\author{
Suraju Olawale Kasali ${ }^{1}$ \\ ${ }^{1}$ Institut Pprime, CNRS, Université de Poitiers, ISAE-ENSMA, F-86962 Futuroscope Chasseneuil, France \\ ${ }^{2}$ Department of Physics/Geology/Geophysics, Alex Ekwueme Federal University Ndufu-Alike Ikwo, Ebonyi State, Nigeria \\ Jose Ordonez-Miranda ${ }^{1, *}$ \\ ${ }^{1}$ Institut Pprime, CNRS, Université de Poitiers, ISAE-ENSMA, F-86962 Futuroscope Chasseneuil, France \\ Karl Joulain ${ }^{1}$ \\ ${ }^{1}$ Institut Pprime, CNRS, Université de Poitiers, ISAE-ENSMA, F-86962 Futuroscope Chasseneuil, France
}

\begin{abstract}
We have theoretically studied and optimized the thermal rectification of plane, cylindrical and spherical radiative thermal diodes operating with terminals of two phase-change materials, whose emissivities significantly change within a narrow interval of temperatures. Analytical expressions for the optimal rectification factors of these three diodes are derived and analyzed comparatively. Optimal rectification factors of $82 \%$, $86 \%$ and $90.5 \%$ are obtained for the plane, cylindrical and spherical diodes made up with terminals of $\mathrm{Ge}_{2} \mathrm{Sb}_{2} \mathrm{Te}_{5}$ and $\mathrm{VO}_{2}$ operating with a temperature difference of $450-300=150 \mathrm{~K}$, respectively. The spherical geometry thus represents a suitable shape to optimize the rectification of radiative heat currents. Furthermore, it is shown that higher rectification factors can potentially be achieved by using phase-change materials with emissivities contrasts higher than those of $\mathrm{Ge}_{2} \mathrm{Sb}_{2} \mathrm{Te}_{5}$ and $\mathrm{VO}_{2}$. We demonstrate that higher rectification factors could be achieved based on the combination of two phase-change materials compared to single phase-change material. The obtained results thus shed light on the phase-change materials required for optimizing the rectification factor of radiative thermal diodes with different geometries.

Keywords: Thermal rectification, heat flows, rectification factor, emissivities, radiative thermal diodes, phase-change materials.
\end{abstract}

\section{INTRODUCTION}

Thermal diodes transporting heat in a preferred direction and blocking it in the opposite one have recently attracted significant attention due to their promising applications on heat control and nanoscale thermal

\footnotetext{
${ }^{*}$ Corresponding Author

Email address: jose.ordonez@cnrs.pprime.fr (Jose Ordonez-Miranda) 
modulation $[1,2,3,4]$. The unusual behavior of thermal diodes have paved the way for controlling the rate of heat flows in a similar way as is done with their electric current counterparts in modern electronic devices. Over the last few years, the rectification effect has been theoretically studied through thermal diodes driven by electrons [5], photons [6, 7, 8, 9, 10], phonons [3, 4, 11, 12, 13], [17, 18], quantum dots, hybrid quantum structures $[14,15,16]$, and nanoporous silicon devices [17]. The experimental study of the diode effect, on the other hand, was done by using quantum dots [19], carbon nanotubes structures [20], graphene nanoribbons [21], nanoporous graphene [22], solid-liquid phase change thermal diodes [23], as well as electrostatic [24] and bulk oxide materials [25].

One of the main methods to rectify heat flows consists of using phase-change materials (PCMs), whose optical, electrical and thermal properties significantly change with temperature, in a relatively narrow interval of temperatures. These changes are generated by the correlated interactions of phonons and electrons inside a PCM [26] and can be applied for developing thermal memories [27], thermal memristors [28, 29], optical switching [30], thermal rectifier [31, 32], thermal switch [33] radiative thermal diodes [34, 35, 36, 37], and radiative thermal transistors [38, 39] operating in both the near- and far-field [40, 34, 38, 26]. This wide variety of promising applications has motivated the interest on PCMs, such as vanadium dioxide $\left(\mathrm{VO}_{2}\right)$, nitinol, $\mathrm{Ge}_{2} \mathrm{Sb}_{2} \mathrm{Te}_{5}$ (GST), whose emissivities can be thermally driven [34, 26, 40]. These three thermochromic materials have recently attracted great attention in both experimental and theoretical studies due to their metal-insulator transition (MIT) at temperatures near room temperature. For example, the emissivities of GST and $\mathrm{VO}_{2}$ undergo the MIT within the temperature ranges of $420 \mathrm{~K}<T<430 \mathrm{~K}$ and $340 \mathrm{~K}<T<345$ $\mathrm{K}$, respectively [41]. The fact that the MIT of $\mathrm{VO}_{2}$ occurs in a temperature interval of only $5 \mathrm{~K}$ has motivated its application as one of the terminals of a radiative thermal diode [34], whose rectification factor can be as high as $90 \%$. This significant rectification is more than four times the typical ones obtained without PCMs [36], which indicates the great potential of these materials to rectify heat flows.

Taking into account that a thermal diode is composed of two terminals and that two PCMs provide more degrees of freedom than a single PCM to tailor the heat transport between them, the rectification factor of radiative thermal diodes is expected to be optimized by the combined effect of two PCMs, as was proposed for conductive thermal diodes [42, 43]. For instance, Yang et al. [44], determined rectification factors up to $50 \%$ and $80 \%$ for a conductive thermal diode made up of two PCMs, whose thermal conductivities vary linearly and quadratically with temperature, respectively. Furthermore, a high rectification ratio of $147 \%$ was also obtained by Kang et al. [45] for a conductive thermal diode operating with two PCMs. These rectification factors are higher than the ones obtained for conductive thermal diodes base on a single PCM $[42,43]$. This fact indicates that two PCMs could also be applied to enhance the rectification factor of radiative thermal diodes, which is not reported in the literature yet, to the best of our knowledge

The goal of this work is to theoretically optimize the thermal rectification effect of plane, cylindrical and spherical radiative thermal diodes based on two PCMs. This is comparatively done by considering the 

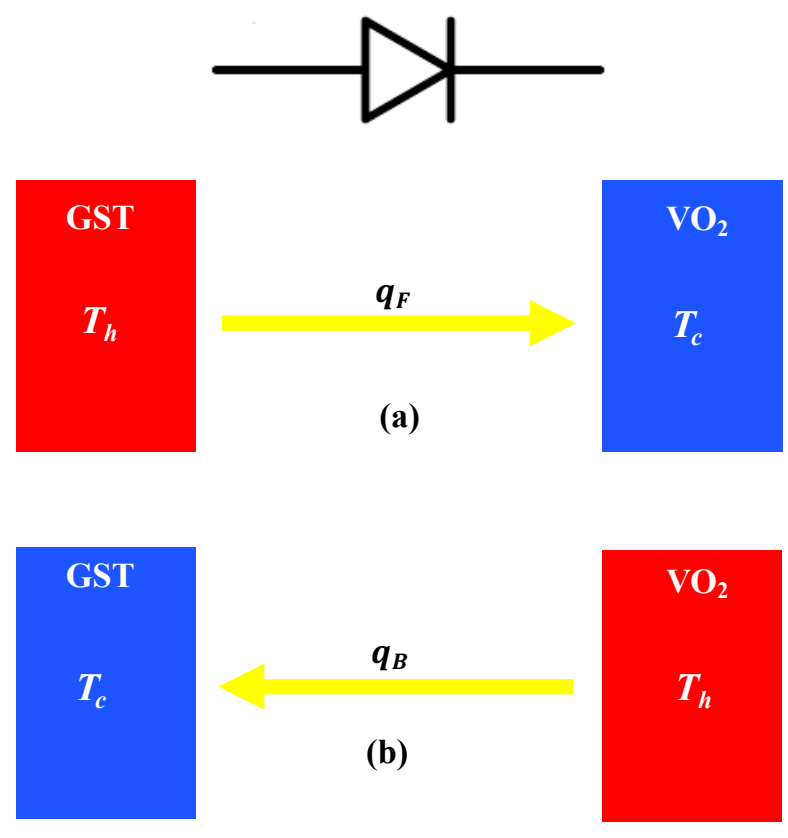

Figure 1: Scheme of a plane radiative thermal diode made up with terminals of GST and $\mathrm{VO}_{2}$ operating in the (a) forward and (b) backward configurations. Hot and cold thermal baths are set at the temperatures $T_{h}$ and $T_{c}$, respectively.

temperature dependence of the emissivities of each PCM within their MITs. Simple analytical expressions are derived and analyzed for the optimal rectification factors of these three diodes supporting thermal radiation generated by a relatively narrow temperature difference between their terminals. Our calculations show that the rectification factor can be raised significantly by using two PCMs instead of the single PCM.

\section{THEORETICAL MODELING}

Let us consider two semi-infinite plates consisting of GST and $\mathrm{VO}_{2}$ exchanging heat through radiation as a result of their temperature difference $T_{h}-T_{c}>0$, as illustrated in Figs. 1(a) and 1(b), respectively. The two plates are separated by a vacuum gap of thickness $d$ much longer than the involved thermal wavelengths to ensure the far-field regime of heat transport. In the forward configuration [Fig. 1(a)], the heat flow $q_{F}$ flows from GST to $\mathrm{VO}_{2}$, while in the backward one [Fig. 1(b)], the heat flow $q_{B}$ flows in the opposite direction due to the interchange of the temperatures $T_{h}$ and $T_{c}$. This behavior of the heat flows $\left(q_{F}\right.$ and $\left.q_{B}\right)$ also holds for the spherical or cylindrical radiative thermal diodes shown in Fig. 2. These heat flows are expected to be different $\left(q_{F} \neq q_{B}\right)$ due to the temperature dependence of the emissivities of GST and $\mathrm{VO}_{2}$, within their MITs. The ability of these temperature-controlled thermal diodes to rectify the heat flows can, therefore, be defined by the following rectification factor $R$ [47]

$$
R=\frac{\left|q_{F}-q_{B}\right|}{\max \left(q_{F}, q_{B}\right)}
$$




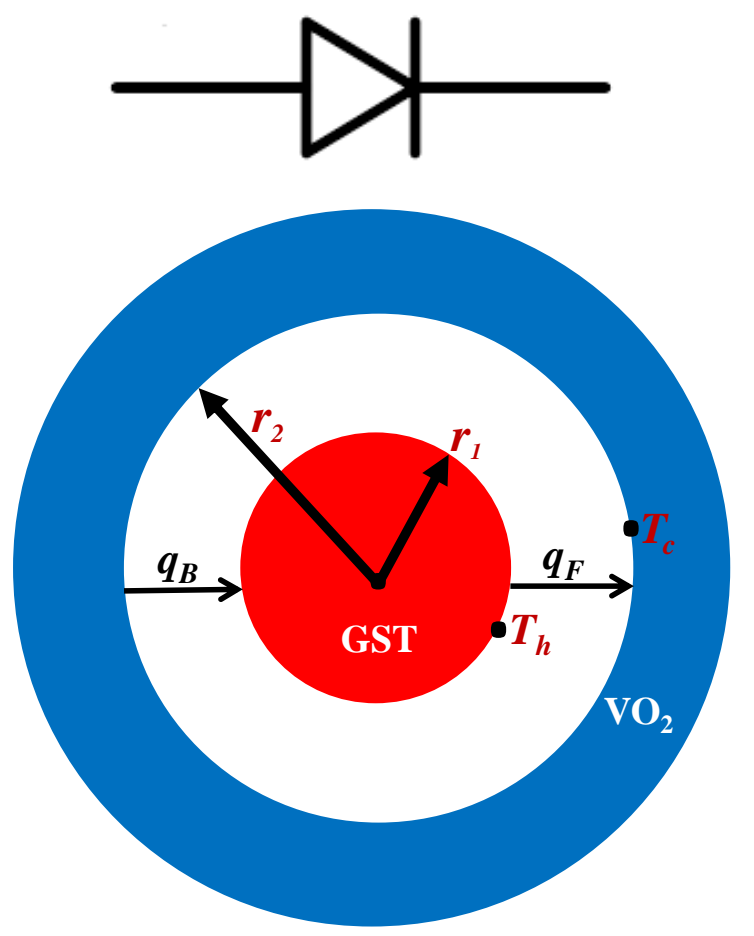

Figure 2: Scheme of a spherical (or cylindrical) radiative thermal diode made up of GST and $\mathrm{VO}_{2}$ operating in the forward and backward configurations. The radii $r_{1}$ and $r_{2}$ are set at the temperatures $T_{h}$ and $T_{c}$ for the forward configuration, while they are interchanged for the backward one. In practice, a spherical diode could be implemented by using the classical Langmuir-Blodgett law [48].

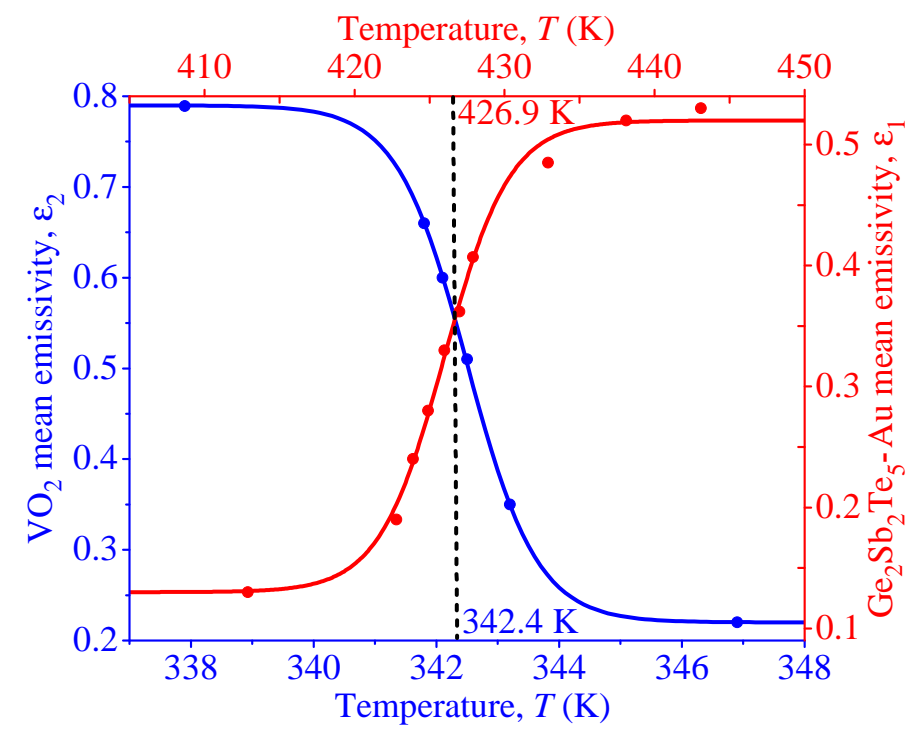

Figure 3: Effective emissivities of GST and $\mathrm{VO}_{2}$ as functions of temperature within their MITs. Dots represent experimental data reported in the literature [43, 46], while lines stand for fits of Eq. (4). 
Equation (1) establishes that a non-zero rectification is reached when the reversal of the thermal gradient induces not only the inversion of the heat flow direction but also a sizeable change of their magnitude. Optimal rectification factor $\left[R_{\mathrm{opt}}=1-q_{B, \min } / q_{F, \max } \rightarrow 1\right]$ can thus be achieved when one of the heat flows is much smaller than the other one. According to Stefan-Boltzmann's law of far-field radiation between two bodies separated by a vacuum gap, the heat flows $q_{F}$ and $q_{B}$ in the forward and backward configurations of the plane, cylindrical and spherical radiative thermal diodes are given by [49]

$$
\begin{aligned}
& q_{F}=A_{1} \sigma \varepsilon_{F}\left(T_{h}^{4}-T_{c}^{4}\right), \\
& q_{B}=A_{2} \sigma \varepsilon_{B}\left(T_{h}^{4}-T_{c}^{4}\right),
\end{aligned}
$$

where $\sigma=5.67 \times 10^{-8} \mathrm{Wm}^{-2} \mathrm{~K}^{-4}$ is the Stefan-Boltzmann constant, $A_{1}$ and $A_{2}$ are the areas of the radiating surfaces, while $\varepsilon_{F}$ and $\varepsilon_{B}$ are their effective emissivities in the forward and backward configurations. The combination of Eqs. (1) and (2) yields the following rectification factor:

$$
R=\frac{\left|A_{1} \varepsilon_{F}-A_{2} \varepsilon_{B}\right|}{\max \left(A_{1} \varepsilon_{F}, A_{2} \varepsilon_{B}\right)} .
$$

Equation (3) explicitly establishes that the values of $R$ are driven by the combined effect of the radiating surfaces and their effective emissivities. The rectification factors of the plane, spherical and cylindrical radiative diodes made up with terminal of GST and $\mathrm{VO}_{2}$ are thus expected to be different due to differences in $A_{1}$ and $A_{2}$. The emissivities of both these PCMs can be described by [50]

$$
\varepsilon_{n}(T)=\varepsilon_{i n}+\frac{\varepsilon_{m n}-\varepsilon_{i n}}{1+e^{-\beta_{n}\left(T-T_{0 n}\right)}},
$$

where $\varepsilon_{m n}$ and $\varepsilon_{i n}$ are the emissivities in their respective high- and low-temperature phases, and $\beta_{n}$ is the phase-transition slope of $\varepsilon_{n}(T)$ at the transition temperature $T=T_{0 n}$, for $n=1$ and 2 . The values of these four parameters were determined by fitting Eq. (4) to the experimental data shown in Fig. 3 for GST and $\mathrm{VO}_{2}$, and they are summarized in Table $\mathrm{I}$.

\begin{tabular}{ccccc}
\multicolumn{5}{c}{ Table 1: Material properties involved in Eq. (4). } \\
\hline \hline Materials & $\varepsilon_{\text {in }}$ & $\varepsilon_{m n}$ & $\beta_{n}\left(\mathrm{~K}^{-1}\right)$ & $T_{0 n}(\mathrm{~K})$ \\
\hline GST & 0.13 & 0.52 & 0.46 & 426.9 \\
$\mathrm{VO}_{2}$ & 0.79 & 0.22 & 1.6 & 342.4 \\
\hline \hline
\end{tabular}

In order to better understand the predictions of Eq. (3) for $R$, its particular and explicit expressions for the plane, cylindrical and spherical radiative diodes are now going to be determined and optimized. 


\subsection{Plane diode}

For the sake of simplicity, we are going to consider that the areas of the radiating surfaces of the plane diode are equal $\left(A_{1}=A_{2}=A\right)$ and therefore the effective emissivities for the forward and backward configurations can be written as follows [49]

$$
\begin{aligned}
& \varepsilon_{F}=\left(\frac{1}{\varepsilon_{1}\left(T_{h}\right)}+\frac{1}{\varepsilon_{2}\left(T_{c}\right)}-1\right)^{-1}, \\
& \varepsilon_{B}=\left(\frac{1}{\varepsilon_{1}\left(T_{c}\right)}+\frac{1}{\varepsilon_{2}\left(T_{h}\right)}-1\right)^{-1},
\end{aligned}
$$

where are $\varepsilon_{n}\left(T_{h}\right)$ and $\varepsilon_{n}\left(T_{c}\right)$ are the emissivities of the PCMs $(n=1 ; 2)$ in their high- and low-temperature phases, respectively. Given that the areas of the radiating surfaces are equal $\left(A_{1}=A_{2}\right)$, the optimal rectification factor in Eq. (3) can be determined by the ratio $\varepsilon_{B \text {, min }} / \varepsilon_{F, \text { max }}$ between the minimum emissivity in the backward configuration to the maximum emissivity in the forward one, as follows:

$$
R_{\mathrm{opt}}=1-\frac{\varepsilon_{B, \min }}{\varepsilon_{F, \max }}
$$

In order to capitalize on the MITs of both PCMs to find $R_{\mathrm{opt}}$, their temperatures should fulfill the condition $T_{c}<T_{0 n}<T_{h}$. According to Fig. (3) and Eqs. (4) and (5), in the forward configuration $\varepsilon_{F}=\varepsilon_{F, \max }$ when the GST terminal is in its high-temperature phase $\left(\varepsilon_{1}\left(T_{h}\right)=\varepsilon_{m 1}\right)$, while the $\mathrm{VO}_{2}$ one is in its lowtemperature phase $\left(\varepsilon_{2}\left(T_{c}\right)=\varepsilon_{i 2}\right)$. In the backward configuration, on the other hand, $\varepsilon_{B}=\varepsilon_{B \text {, min }}$ when the GST terminal is in its low-temperature phase $\left(\varepsilon_{1}\left(T_{c}\right)=\varepsilon_{i 1}\right)$, while the $\mathrm{VO}_{2}$ one is in its high-temperature phase $\left(\varepsilon_{2}\left(T_{h}\right)=\varepsilon_{m 2}\right)$. Under these conditions, Eqs. (5a) and (5b) reduce to

$$
\begin{aligned}
& \varepsilon_{F, \max }=\left(\frac{1}{\varepsilon_{m 1}}+\frac{1}{\varepsilon_{i 2}}-1\right)^{-1} \\
& \varepsilon_{B, \min }=\left(\frac{1}{\varepsilon_{i 1}}+\frac{1}{\varepsilon_{m 2}}-1\right)^{-1}
\end{aligned}
$$

After inserting Eqs. (7a) and (7b) into Eq. (6), the following optimal rectification factor $R_{\text {opt }}$ of a radiative plane diode is obtained

$$
R_{\mathrm{opt}}=1-\frac{\varepsilon_{m 2}}{\varepsilon_{i 2}} \frac{\left(1+\varepsilon_{i 2} / \varepsilon_{m 1}-\varepsilon_{i 2}\right)}{\left(1+\varepsilon_{m 2} / \varepsilon_{i 1}-\varepsilon_{m 2}\right)} .
$$

Note that $R_{\text {opt }}$ is symmetric on the emissivities of both PCMs and increases with the ratios $\varepsilon_{i 1} / \varepsilon_{m 1}$ and $\varepsilon_{i 2} / \varepsilon_{m 2}$ of GST and $\mathrm{VO}_{2}$, respectively. Materials with a higher emissivity contrasts are thus expected to yield radiative diodes with higher rectification factors, as expected. 


\subsection{Cylindrical diode}

In this case, the areas $A_{1}$ and $A_{2}$ of the radiation surfaces depends on their respective radii $r_{1}$ and $r_{2}$, such that $A_{1} / A_{2}=r_{1} / r_{2}$. The effective emissivities for the forward and backward configurations are hence given by [49]

$$
\begin{aligned}
& \varepsilon_{F}=\left(\frac{1}{\varepsilon_{1}\left(T_{h}\right)}+\frac{1-\varepsilon_{2}\left(T_{c}\right)}{\varepsilon_{2}\left(T_{c}\right)}\left(\frac{r_{1}}{r_{2}}\right)\right)^{-1}, \\
& \varepsilon_{B}=\left(\frac{1}{\varepsilon_{1}\left(T_{c}\right)}+\frac{1-\varepsilon_{2}\left(T_{h}\right)}{\varepsilon_{2}\left(T_{h}\right)}\left(\frac{r_{2}}{r_{1}}\right)\right)^{-1} .
\end{aligned}
$$

where $r_{1}$ and $r_{2}$ are radii of the cylindrical radiating surfaces (Fig. 2). In contrast to the corresponding effective emissivities for the plane diode, Eqs. (9a) and (9b) do depends on ratio $r_{1} / r_{2}$, which provides another degree of freedom to optimize the rectification factor of the radiative cylindrical diode. In this case, Eq. (3) takes the form

$$
R_{\mathrm{opt}}=1-\frac{r_{2} \varepsilon_{B, \min }}{r_{1} \varepsilon_{F, \max }},
$$

where the effective emissivities $\varepsilon_{F}=\varepsilon_{F, \max }$ and $\varepsilon_{B}=\varepsilon_{B \text {,min }}$ for the forward and backward configurations can be found by following a similar procedure than the one developed for the plane diode. The final results are

$$
\begin{aligned}
& \varepsilon_{F, \max }=\left(\frac{1}{\varepsilon_{m 1}}+\frac{1-\varepsilon_{i 2}}{\varepsilon_{i 2}}\left(\frac{r_{1}}{r_{2}}\right)\right)^{-1}, \\
& \varepsilon_{B, \text { min }}=\left(\frac{1}{\varepsilon_{i 1}}+\frac{1-\varepsilon_{m 2}}{\varepsilon_{m 2}}\left(\frac{r_{2}}{r_{1}}\right)\right)^{-1} .
\end{aligned}
$$

The optimal rectification factor $R_{\mathrm{opt}}$ of the cylindrical diode is then determined by the combination of Eqs. (10) and (11), as follows

$$
R_{\mathrm{opt}}=1-\frac{\varepsilon_{m 2}}{\varepsilon_{i 2}}\left(\frac{r_{1}}{r_{2}}\right) \frac{\left(1+r_{2} \varepsilon_{i 2} / r_{1} \varepsilon_{m 1}-\varepsilon_{i 2}\right)}{\left(1+r_{1} \varepsilon_{m 2} / r_{2} \varepsilon_{i 1}-\varepsilon_{m 2}\right)} .
$$

The comparison of Eqs. (8) and (12) indicates that the values of $R_{\mathrm{opt}}$ for a cylindrical diode could be higher than the corresponding ones for a plane diode, by properly modulating the ratio $r_{1} / r_{2}$ as well as the emissivity contrast of the PCMs.

\subsection{Spherical diode}

In this case, the ratio of the areas $A_{1}$ and $A_{2}$ of the radiating surfaces is given by the square of the one of their radii $\left(A_{1} / A_{2}=\left(r_{1} / r_{2}\right)^{2}\right)$ and the effective emissivities for the forward and backward configurations 
are given by $[49]$

$$
\begin{aligned}
& \varepsilon_{F}=\left(\frac{1}{\varepsilon_{1}\left(T_{h}\right)}+\frac{1-\varepsilon_{2}\left(T_{c}\right)}{\varepsilon_{2}\left(T_{c}\right)}\left(\frac{r_{1}}{r_{2}}\right)^{2}\right)^{-1}, \\
& \varepsilon_{B}=\left(\frac{1}{\varepsilon_{1}\left(T_{c}\right)}+\frac{1-\varepsilon_{2}\left(T_{h}\right)}{\varepsilon_{2}\left(T_{h}\right)}\left(\frac{r_{2}}{r_{1}}\right)^{2}\right)^{-1},
\end{aligned}
$$

Equations (13a) and (13b) are similar to Eqs. (9a) and (9b) for the cylindrical diode, but the only difference is that the ratio $r_{1} / r_{2}$ is now squared. The spherical geometry is thus expected to have a different impact than the cylindrical one on the rectification factor of a radiative diode. According to Eq. (3), the optimal rectification factor can be written in terms of the effective emissivities for the spherical diode as follows:

$$
R_{\mathrm{opt}}=1-\frac{r_{2}^{2} \varepsilon_{B, \min }}{r_{1}^{2} \varepsilon_{F, \max }},
$$

where, according to Eqs. (13a) and (13b), the effective emissivities $\varepsilon_{F}=\varepsilon_{F, \text { max }}$ and $\varepsilon_{B}=\varepsilon_{B \text {,min }}$ for the forward and backward configurations are given by

$$
\begin{aligned}
& \varepsilon_{F}, \max =\left(\frac{1}{\varepsilon_{m 1}}+\frac{1-\varepsilon_{i 2}}{\varepsilon_{i 2}}\left(\frac{r_{1}}{r_{2}}\right)^{2}\right)^{-1}, \\
& \varepsilon_{B}, \min =\left(\frac{1}{\varepsilon_{i 1}}+\frac{1-\varepsilon_{m 2}}{\varepsilon_{m 2}}\left(\frac{r_{1}}{r_{2}}\right)^{2}\right)^{-1} .
\end{aligned}
$$
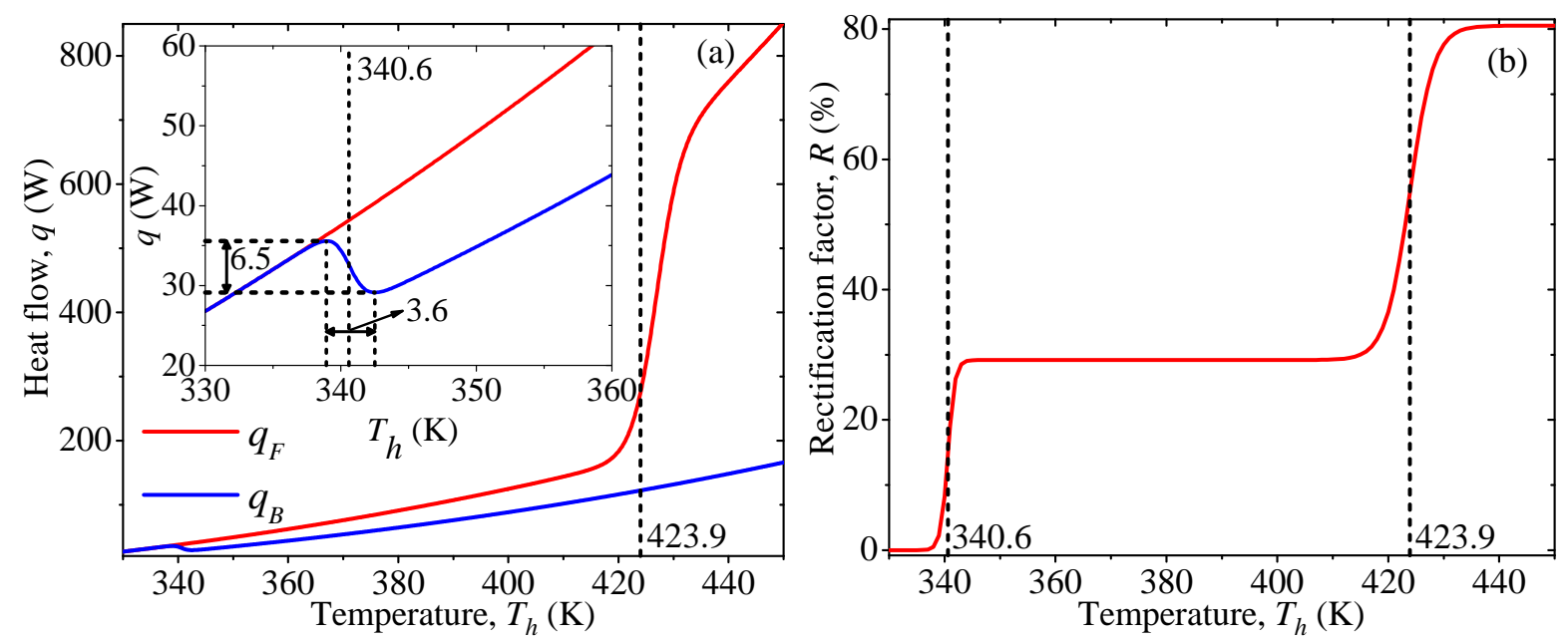

Figure 4: Temperature dependence of the radiative heat flows for the (a) forward and backward configurations of a plane radiative diode operating with terminals of GST and $\mathrm{VO}_{2}$. Inset: Zoom-in of the first maximum and minimum values of $q_{B}$. (b) Rectification factor as a function of $T_{h}$. Calculations were done for $T_{c}=300 \mathrm{~K}$.

Finally, the explicit expression for $R_{\mathrm{opt}}$ is determined by the combination of Eqs. (14) and (15), which 

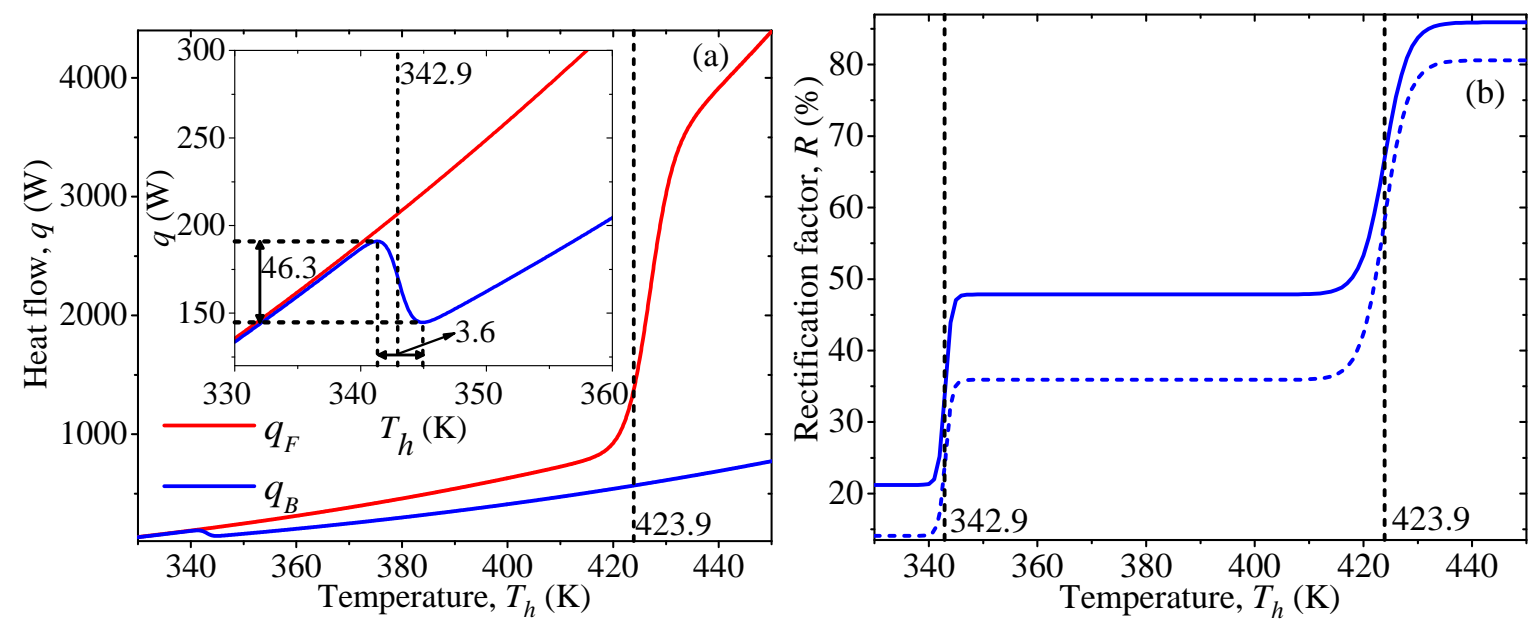

Figure 5: Temperature dependence of the radiative heat flows for the (a) forward and backward configurations of a cylindrical radiative diode operating with terminals of GST and $\mathrm{VO}_{2}$ along with (b) its corresponding rectification factor. The solid line stand for the thermal diode shown in Fig. 2, while the dashed one corresponds to a system with interchanged terminals. The inset in (a) shows a zoom-in of the first maximum and minimum values of $q_{B}$. Calculations were done for $T_{c}=300 \mathrm{~K}$ and $r_{1} / r_{2}=0.8$.

yield

$$
R_{\mathrm{opt}}=1-\frac{\varepsilon_{m 2}}{\varepsilon_{i 2}}\left(\frac{r_{1}}{r_{2}}\right)^{2} \frac{\left(1+r_{2}^{2} \varepsilon_{i 2} / r_{1}^{2} \varepsilon_{m 1}-\varepsilon_{i 2}\right)}{\left(1+r_{1}^{2} \varepsilon_{m 2} / r_{2}^{2} \varepsilon_{i 1}-\varepsilon_{m 2}\right)} .
$$

One can see that $R_{\text {opt }}$ is symmetrical on the emissivities of both PCMs and has a stronger (squared) dependence on the ratio $r_{1} / r_{2}$ than that of a cylindrical diode. The spherical diode is thus expected to yield higher rectification factors than the corresponding cylindrical one, as shown below.

\section{RESULTS AND DISCUSSIONS}

Figure 4(a) shows the heat flows exchanged by the terminals of a plane diode operating in the forward and backward configurations, as functions of the temperature $T_{h}$. Note that both heat flows $q_{F}$ and $q_{B}$ exhibit a nearly linear increase with $T_{h}$ and change of slope at temperatures around the transition temperatures $\left(T_{h} \approx T_{0 n}\right.$ with $\left.n=1,2\right)$ of the PCMs emissivities. According to Fig. 3, the transition of $q_{B}$ at $T_{h}=340.6$ $\mathrm{K}$ is related to the MIT of $\mathrm{VO}_{2}$, while that of $q_{F}$ at $T_{h}=343.9 \mathrm{~K}$ is driven by the MIT of GST. For $T_{h}<340 \mathrm{~K}$, both PCMs do not undergo any phase transition, $q_{F}$ and $q_{B}$ are equal, and therefore there is no rectification, as shown in Fig. 4(b). By contrast, the rectification factor reaches its first plateau $R \simeq 30 \%$ just above the $\mathrm{VO}_{2} \operatorname{MIT}\left(T_{h}>340 \mathrm{~K}\right)$. For higher temperatures $\left(T_{h}>423.9 \mathrm{~K}\right)$, both terminals undergo their MITs and the rectification factor increases to $R \simeq 82 \%$, which agrees with the predictions of Eq. (8). This fact indicates that the combination of the MITs of GST and $\mathrm{VO}_{2}$ is able to maximize the rectification factor of a plane diode to values higher than the corresponding ones obtained with a single PCM [43, 35]. 

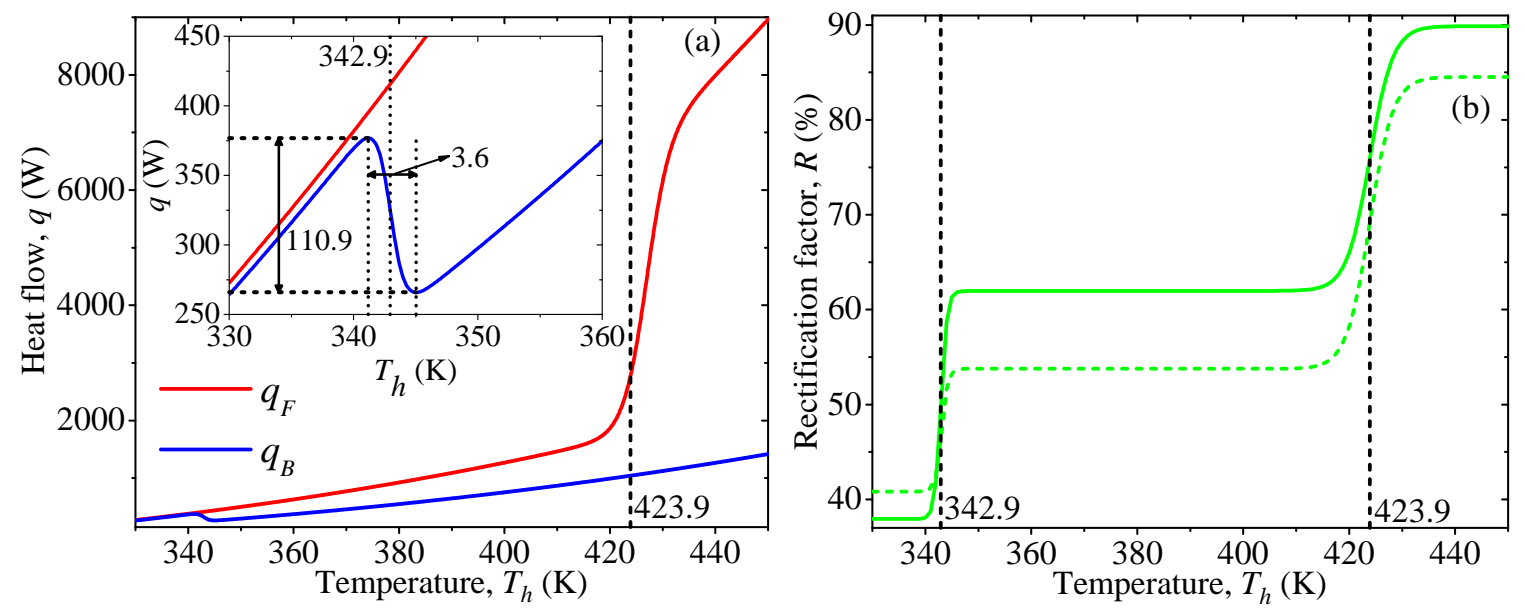

Figure 6: Temperature dependence of the radiative heat flows for the (a) forward and backward configurations of a spherical radiative diode operating with terminals of GST and $\mathrm{VO}_{2}$ along with (b) its corresponding rectification factor. The solid line stand for the thermal diode shown in Fig. 2, while the dashed one corresponds to a system with interchanged terminals. The inset in (a) shows a zoom-in of the first maximum and minimum values of $q_{B}$. Calculations were done for $T_{C}=300 \mathrm{~K}$ and $r_{1} / r_{2}=0.8$

The temperature dependence of the heat flows $q_{F}$ and $q_{B}$ for the forward and backward configurations of a cylindrical diode along with its rectification factor are shown in Figs. 5(a) and 5(b), respectively. Note that the values of the heat flow generated in the cylindrical diode exhibits a similar behavior than the corresponding ones in the plane diode, although their values are relatively higher than these latter ones. The diode geometry has thus a significant impact on the values of the heat flows, whose behavior is determined by the PCMs' emissivities. One can see that both $q_{F}$ and $q_{B}$ increase linearly with $T_{h}$, such that their difference is greater than zero for temperatures $T_{h} \leq 342.9 \mathrm{~K}$. The sharp increase of $q_{F}$ around $T_{h}=423.9$ $\mathrm{K}$ is generated by the GST emissivity and allows increasing the rectification factor up to $R \simeq 86 \%$. This value is consistent with Eq. (12) and is higher than the one obtained for a plane diode (Fig. 4(b)).

The heat flows $q_{F}$ and $q_{B}$ of a spherical diode operating with terminals of GST and $\mathrm{VO}_{2}$ are shown in Fig. 6(a). Note that the linear increase of $q_{F}$ and $q_{B}$ with $T_{h}$ is consistent with the one reported for the plane and cylindrical diodes, such that their values are relatively higher than the corresponding ones for these two latter diodes. Note also that the temperature interval (3.6 K in width) between the first maximum and first minimum of $q_{B}$ of the three diodes is the same, which confirms that the behavior of the heat flux is driven by the PCMs' emissivities. Taking into account that these extreme values of $q_{B}$ appear at temperatures close to the those determining the borders of the metal-insulator transition of $\mathrm{VO}_{2}$ (Fig. 3), the local maximum of $q_{B}$ is related to the appearance of this phase transition, while the local minimum of $q_{B}$ is associated with its disappearance, as the temperature is raised. On the other hand, the two plateaux of the rectification factor occur at $R \simeq 63 \%$ and $R \simeq 90.5 \%$ (Fig. 6(b)), which are higher than the corresponding ones of the 


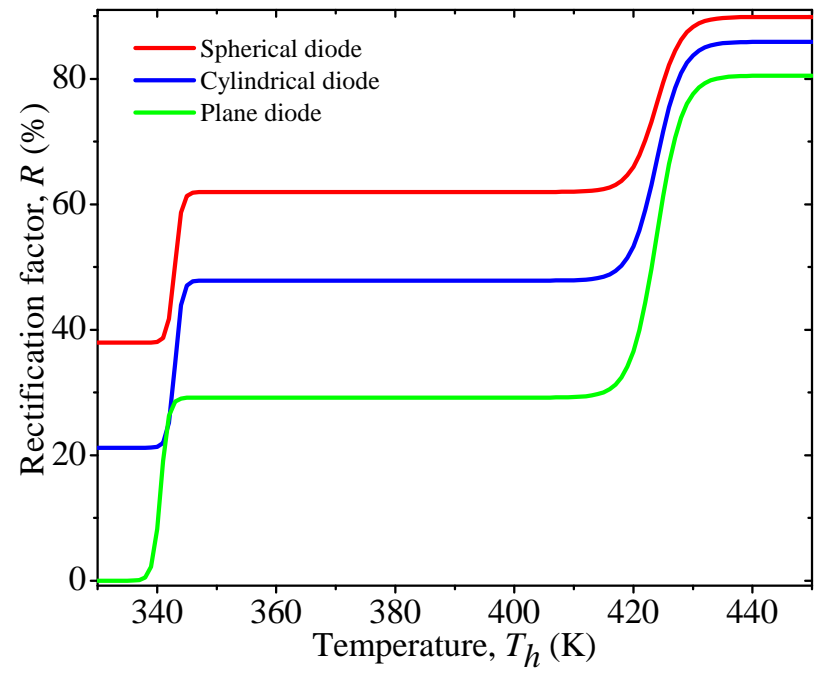

Figure 7: Comparison of the rectification factors obtained for the plane, cylindrical, and spherical diodes operating with terminals of GST and $\mathrm{VO}_{2}$. Calculations were done for $T_{c}=300 \mathrm{~K}$ and $r_{1} / r_{2}=0.8$.
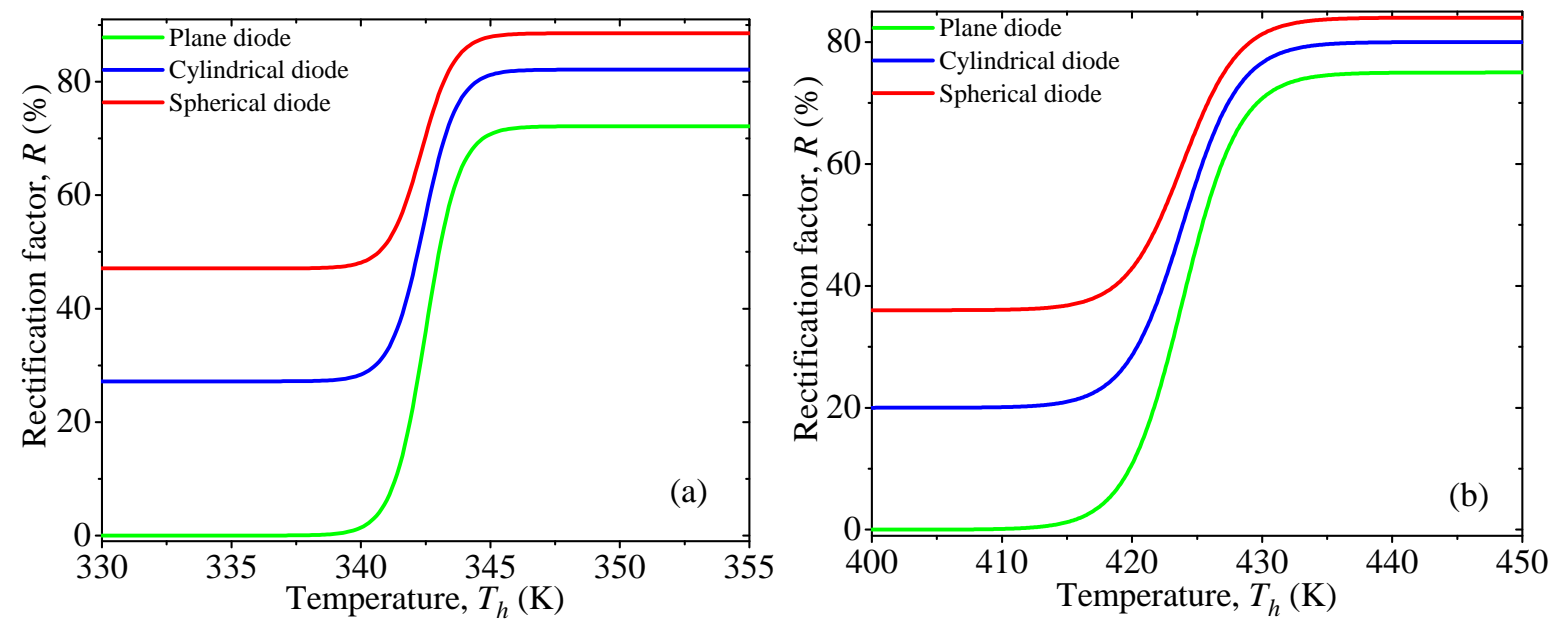

Figure 8: Temperature dependence of the rectification factor of plane, cylindrical, and spherical diodes with terminals of (a) $\mathrm{VO}_{2}$ and a black body and (b) GST and black body. Calculations were done for $T_{c}=300 \mathrm{~K}$ and $r_{1} / r_{2}=0.8$. 

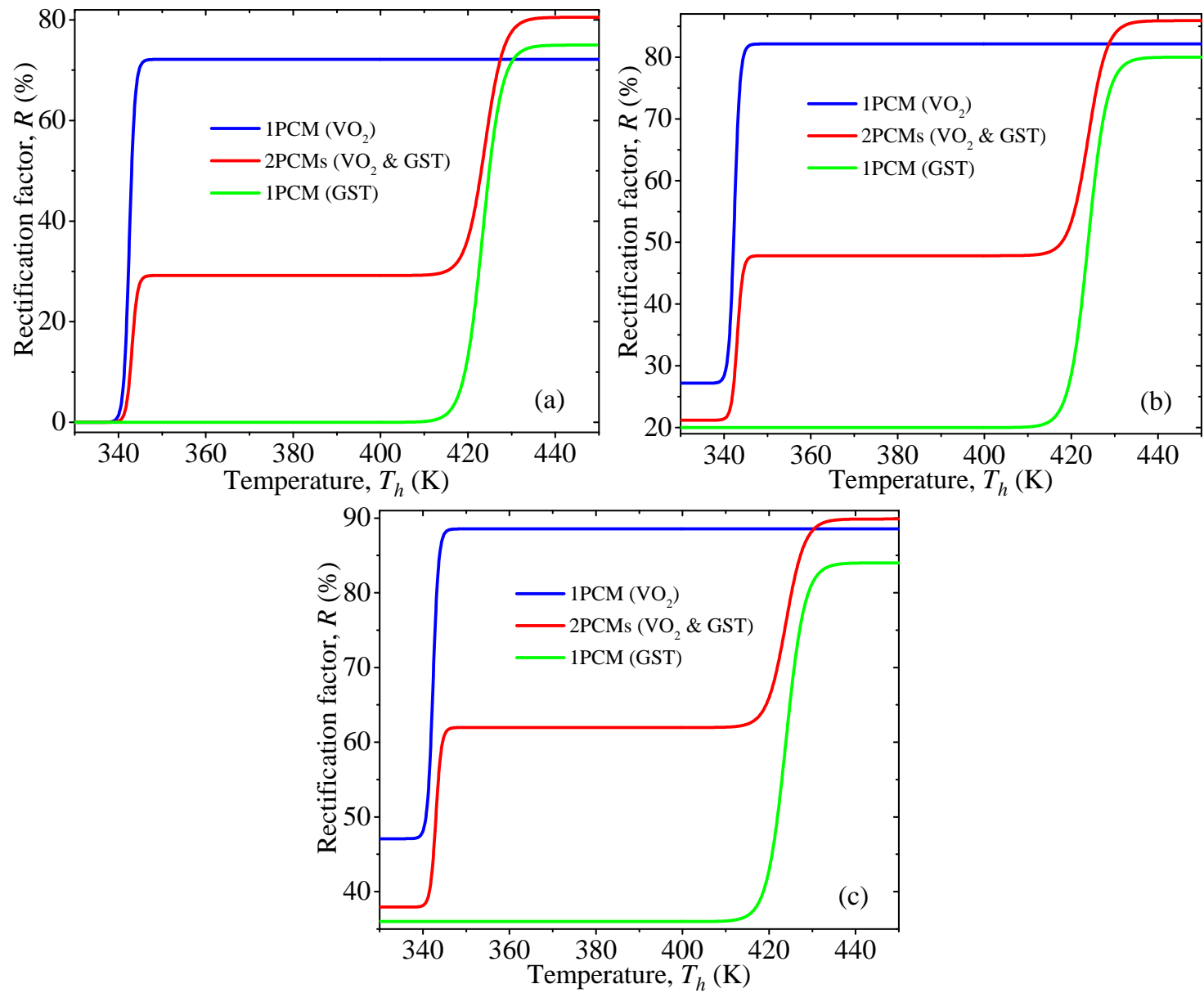

Figure 9: Rectification factors of (a) plane (b) cylindrical and (c) spherical diodes based on one PCM and two PCMs, as functions of $T_{h}$. Calculations were done for $T_{c}=300 \mathrm{~K}$ and $r_{1} / r_{2}=0.8$.

plane and cylindrical diodes. This fact indicates that the spherical geometry is better than the other two diode geometries to maximize the rectification of radiative heat flows, as shown in Fig. 7. Note that if the terminals of the thermal diode shown in Fig. 2 are interchanged, the rectification factors of the cylindrical and spherical thermal diodes are smaller than the corresponding ones obtained for the system in Fig. 2, as shown in Figs. 5(b) and 6(b). This is expected given that both diode geometries have a strong dependence on the effective emissivities ratio and area of the radiating surfaces. However, this is not the case for the plane diode rectification factor, as the areas of the radiating surfaces are equal.

In order to single out the effect of a PCM on the rectification factor of the three radiative diodes under consideration, we have replaced the GST terminal by a black body (emissivity $=1$ ) and plotted the temperature dependence of the rectification factors thus obtained in Fig. 8(a). Note that $R$ undergoes a single transition generated by the $\mathrm{VO}_{2} \mathrm{MIT}$, such that it reaches a plateau for high enough temperatures. A similar behavior is observed when the $\mathrm{VO}_{2}$ terminal is replaced by a black body, as shown in Fig. 8(b). In 
any case, the maximum rectification factors obtained with a single PCM are lower than the corresponding ones obtained with two PCMs, as shown in Figs. 9(a), 9(b), and 9(c) for the plane, cylindrical and spherical radiative diodes, respectively. In fact, the relative increase of the maximum rectification factors obtained with $2 \mathrm{PCMs}$ and $1 \mathrm{PCM}$ are $82-75=7 \%, 86-80=6 \%$, and $90.5-84=6.5 \%$ for the plane, cylindrical and spherical diodes, respectively. These similar values indicate that, with a given geometry, the improvement of the rectification factor is due to the phase transitions of both PCMs mainly. This study thus provides an analytical theoretical model to describe and optimize the rectification factor of radiative thermal diodes operating with two PCMs of different geometries. The obtained results therefore allow obtaining rectification factors higher than those generated by a single PCM [6, 7, 35, 34].

\section{CONCLUSION}

We have theoretically analyzed and optimized the rectification factors of plane, cylindrical and spherical radiative thermal diodes with terminals made up of two phase-change materials. This has been done by deriving analytical expressions for the optimal rectification factors of these three diodes. It has been shown that combined effect of two phase-change materials along with the geometry of the thermal diode has a significant impact on its rectification factor. Optimal rectification factors of $82 \%, 86 \%$ and $90.5 \%$ have been determined for the plane, cylindrical, and spherical diodes operating with terminals of $\mathrm{Ge}_{2} \mathrm{Sb}_{2} \mathrm{Te}_{5}$ and $\mathrm{VO}_{2}$ with a temperature difference up to $150 \mathrm{~K}$. The obtained rectification factors indicate that the combination of two phase-change materials is rather high compared to previous radiative thermal diodes concepts based on single phase-change material. The highest rectification of radiative heat flows is thus obtained for the spherical diode and the lowest one for the plane diode. These high rectification factors could become even higher in a narrower interval of temperatures by using phase-change materials with greater emissivity variations and faster phase transitions than the corresponding ones of $\mathrm{Ge}_{2} \mathrm{Sb}_{2} \mathrm{Te}_{5}$ and $\mathrm{VO}_{2}$. The obtained results can thus guide the development of phase-change materials capable of optimizing the rectification factors of radiative thermal diodes with different geometries.

\section{References}

[1] T.-M. Shih, Z. Gao, Z. Guo, H. Merlitz, P. J. Pagni, Z. Chen, Maximal rectification ratios for idealized bi-segment thermal rectifiers, Sci. Rep. 5 (2015) 12677.

[2] N. Li, J. Ren, L. Wang, G. Zhang, P. Hänggi, B. Li, Colloquium: Phononics: Manipulating heat flow with electronic analogs and beyond, Rev. Mod. Phys. 84 (2012) 1045.

[3] N. A. Roberts, D. Walker, A review of thermal rectification observations and models in solid materials, Int. J. Therm. Sci. 50 (2011) 648.

[4] B. Li, L. Wang, G. Casati, Thermal diode: Rectification of heat flux, Phys. Rev. Lett. 93 (2004) 184301.

[5] D. Segal, Single mode heat rectifier: Controlling energy flow between electronic conductors, Phys. Rev. Lett. 100 (2008) 105901. 
[6] C. R. Otey, W. T. Lau, S. Fan, et al., Thermal rectification through vacuum, Phys. Rev. Lett. 104 (2010) 154301.

[7] H. Iizuka, S. Fan, Rectification of evanescent heat transfer between dielectric-coated and uncoated silicon carbide plates, J. Appl. Phys. 112 (2012) 024304.

[8] S. Basu, M. Francoeur, Near-field radiative transfer based thermal rectification using doped silicon, Appl. Phys. Lett. 98 (2011) 113106.

[9] L. Wang, Z. Zhang, Thermal rectification enabled by near-field radiative heat transfer between intrinsic silicon and a dissimilar material, Nanosc. Microsc. Therm. 17 (2013) 337.

[10] K. Joulain, Y. Ezzahri, J. Drevillon, B. Rousseau, D. D. S. Meneses, Radiative thermal rectification between sic and sio 2, Opt. Express 23 (2015) A1388.

[11] K. Garcia-Garcia, J. Alvarez-Quintana, Thermal rectification assisted by lattice transitions, Int. J. Therm. Sci. 81 (2014) 76.

[12] G. Zhang, H. Zhang, Thermal conduction and rectification in few-layer graphene y junctions, Nanoscale 3 (2011) 4604.

[13] E. Pereira, Sufficient conditions for thermal rectification in general graded materials, Phys. Rev. E 83 (2011) 031106.

[14] J. Ordonez-Miranda, Y. Ezzahri, K. Joulain, Quantum thermal diode based on two interacting spinlike systems under different excitations, Phys. Rev. E 95 (2017) 022128.

[15] L.-A. Wu, D. Segal, Sufficient conditions for thermal rectification in hybrid quantum structures, Phys. Rev. Lett. 102 (2009) 095503.

[16] T. Ruokola, T. Ojanen, A.-P. Jauho, Thermal rectification in nonlinear quantum circuits, Phys. Rev. B 79 (2009) 144306.

[17] H. Machrafi, G. Lebon, D. Jou, Thermal rectifier efficiency of various bulk-nanoporous silicon devices, Int. J. Heat Mass Trans. 97 (2015) 603-610.

[18] M. Criado-Sancho, LF. Del Castillo, J. Casas-Vázquez, D. Jou, Theoretical analysis of thermal rectification in a bulk $\mathrm{Si} /$ nanoporous Si device, Phys. Lett. A 376 (19) (2012) 1641-1644.

[19] R. Scheibner, M. König, D. Reuter, A. Wieck, C. Gould, H. Buhmann, L. Molenkamp, Quantum dot as thermal rectifier, New J. Phys. 10 (8) (2008) 083016.

[20] G. Wu, B. Li, Thermal rectification in carbon nanotube intramolecular junctions: Molecular dynamics calculations, Phys. Rev. B 76 (8) (2007) 085424.

[21] K. Yuan, M. Sun, Z. Wang, D. Tang, Tunable thermal rectification in silicon-functionalized graphene nanoribbons by molecular dynamics simulation, Int. J. Therm. Sci. 98 (2015) 24-31.

[22] F. Yousefi, F. Khoeini, A. Rajabpour, Thermal conductivity and thermal rectification of nanoporous graphene: A molecular dynamics simulation, Int. J. Heat Mass Trans. 146 (2020) 118884.

[23] Z. Meng, R. Gulfam, P. Zhang, F. Ma, Numerical and experimental study of the thermal rectification of a solid-liquid phase change thermal diode, Int. J. Heat Mass Trans. 147 (2020) 118915.

[24] B. Traipattanakul, CY. Tso, CYH. Chao, A phase-change thermal diode using electrostatic-induced coalescing-jumping droplets, Int. J. Heat Mass Trans. 135 (2019) 294-304.

[25] W. Kobayashi, Y. Teraoka, I. Terasaki, An oxide thermal rectifier, Appl. Phys. Lett. 95 (17) (2009) 171905.

[26] K. Joulain, Y. Ezzahri, J. Drevillon, P. Ben-Abdallah, Modulation and amplification of radiative far field heat transfer: Towards a simple radiative thermal transistor, Appl. Phys. Lett. 106 (2015) 133505.

[27] K. Ito, K. Nishikawa, H. Iizuka, Multilevel radiative thermal memory realized by the hysteretic metal-insulator transition of vanadium dioxide, Appl. Phys. Lett. 108 (2016) 053507.

[28] P. Ben-Abdallah, Thermal memristor and neuromorphic networks for manipulating heat flow, AIP Adv. 7 (2017) 065002.

[29] J. Ordonez-Miranda, Y. Ezzahri, J. A. Tiburcio-Moreno, K. Joulain, J. Drevillon, Radiative thermal memristor, Phys. Rev. Lett. 123 (2019) 025901.

[30] M. Rini, Z. Hao, R. Schoenlein, C. Giannetti, F. Parmigiani, S. Fourmaux, J. Kieffer, A. Fujimori, M. Onoda, S. Wall, 
Optical switching in vo 2 films by below-gap excitation, Appl. Phys. Lett. 92 (18) (2008) 181904.

[31] MG. Naso, E. Vuk, F. Zullo, On the optimization of heat rectification in graded materials, Int. J. Heat Mass Trans. 143 (2019) 118520.

[32] J. Huang, Q. Li, Z. Zheng, Y. Xuan, Thermal rectification based on thermochromic materials, Int. J. Heat Mass Trans. 67 (2013) 575-580

[33] W. Gu, GH. Tang, WQ. Tao, Thermal switch and thermal rectification enabled by near-field radiative heat transfer between three slabs, Int. J. Heat Mass Trans. 82 (2015) 429-434.

[34] P. Ben-Abdallah, S.-A. Biehs, Phase-change radiative thermal diode, Appl. Phys. Lett. 103 (2013) 191907.

[35] E. Nefzaoui, K. Joulain, J. Drevillon, Y. Ezzahri, Radiative thermal rectification using superconducting materials, Appl. Phys. Lett. 104 (2014) 103905.

[36] E. Nefzaoui, J. Drevillon, Y. Ezzahri, K. Joulain, Simple far-field radiative thermal rectifier using fabry-perot cavities based infrared selective emitters, Appl. Opt. 53 (2014) 3479.

[37] K. Ito, K. Nishikawa, H. Iizuka, H. Toshiyoshi, Experimental investigation of radiative thermal rectifier using vanadium dioxide, Appl. Phys. Lett. 105 (2014) 253503.

[38] P. Ben-Abdallah, S.-A. Biehs, Near-field thermal transistor, Phys. Rev. Lett. 112 (2014) 044301.

[39] H. Prod'homme, J. Ordonez-Miranda, Y. Ezzahri, J. Drevillon, K. Joulain, Optimized thermal amplification in a radiative transistor, Journal of Applied Physics 119 (2016) 194502.

[40] P. J. van Zwol, L. Ranno, J. Chevrier, Emissivity measurements with an atomic force microscope, J. Appl. Phys. 111 (2012) 063110.

[41] A. Barker Jr, H. Verleur, H. Guggenheim, Infrared optical properties of vanadium dioxide above and below the transition temperature, Phys. Rev. Lett. 17 (1966) 1286.

[42] A. L. Cottrill, M. S. Strano, Analysis of thermal diodes enabled by junctions of phase change materials, Adv. Energy Mater. 5 (23) (2015) 1500921.

[43] J. Ordonez-Miranda, J. M. Hill, K. Joulain, Y. Ezzahri, J. Drevillon, Conductive thermal diode based on the thermal hysteresis of vo2 and nitinol, J. Appl. Phys. 123 (2018) 085102.

[44] Y. Yang, H. Chen, H. Wang, N. Li, L. Zhang, Optimal thermal rectification of heterojunctions under fourier law, Phys. Rev. E 98 (4) (2018) 042131.

[45] H. Kang, F. Yang, J. J. Urban, Thermal rectification via heterojunctions of solid-state phase-change materials, Phys. Rev. Appl. 10 (2) (2018) 024034.

[46] K.-K. Du, Q. Li, Y.-B. Lyu, J.-C. Ding, Y. Lu, Z.-Y. Cheng, M. Qiu, Control over emissivity of zero-static-power thermal emitters based on phase-changing material gst, Light Sci. Appl. 6 (2017) e16194.

[47] S. O. Kasali, J. Ordonez-Miranda, K. Joulain, Spherical and cylindrical conductive thermal diodes based on vo2, Eur. Phys. J. Plus 134 (7) (2019) 340.

[48] I. Langmuir, K. B. Blodgett, Currents limited by space charge between concentric spheres, Phys. Rev. 24 (1924) 49-59.

[49] F. P. Incropera, D. P. DeWitt, Fundamentals of heat and mass transfer, Vol. 4, Wiley New York, 1996.

[50] J. Ordonez-Miranda, K. Joulain, Y. Ezzahri, J. Drevillon, J. Alvarado-Gil, Periodic amplification of radiative heat transfer, J. Appl. Phys. 125 (2019) 064302. 